\title{
Hidroquímica do rio Solimões na região entre Manacapuru e Alvarães - Amazonas - Brasil
}

\begin{abstract}
Maria Mireide Andrade QUEIROZ ${ }^{1}$, Adriana Maria Coimbra HORBE², Patrick SEYLER ${ }^{3}$, Candido Augusto Veloso MOURA ${ }^{4}$

RESUMO

Este trabalho discute as características físico-químicas das águas dos rios Solimões, Purus e seus afluentes, coletadas em novembro de 2004 no Estado do Amazonas, entre as cidades de Manacapuru-Alvarães e Anamã-Pirarauara. Foram realizadas análises físico-químicas (temperatura, $\mathrm{pH}$, condutividade elétrica, turbidez, $\mathrm{Ca}^{2+}, \mathrm{Na}^{+}, \mathrm{K}^{+}, \mathrm{Mg}^{2+}, \mathrm{HCO}_{3}^{-}, \mathrm{SO}_{4}^{2-}, \mathrm{Cl}^{-}$), de elementostraço (Li, B, Al, Sc, V, Cr, Mn, Fe, Co, Cu, Zn, As, Se, Rb, Sr, Mo, Cd, Sb, Cs, Ba, Pb, La, Ce e U) e isótopos de estrôncio. Os parâmetros analisados e a composição química mostram que as águas dos rios e igarapés da região central da Amazônia são quimicamente distintas entre si. As águas brancas do Solimóes são cálcicas-bicarbonatadas e as do Purus bicarbonatadas, os respectivos afluentes são sódico-potássico-bicarbonatados e sódico-potássico-sulfatados. Isso acarreta águas brancas fracamente ácidas a neutras e mais condutivas, enquanto as pretas são menos mineralizadas, mais ácidas, especialmente as do Purus. O Ba, $\mathrm{Sr}, \mathrm{Cu}, \mathrm{V}$ e As mais elevados diferenciam as águas brancas do Solimóes das do Purus, bem como os afluentes do primeiro em relação ao segundo. Esse conjunto de características indicam que tanto o Solimóes, como o Purus e os respectivos afluentes, estão submetidos a condiçóes geológicas/ambientais distintas. A influência do aporte de sedimentos dos Andes é diluída ao longo da bacia do Solimóes e se reflete na formaçáo das várzeas dos Solimóes e Purus. Por outro lado as rochas crustais, representadas pelos escudos das Guianas e Brasileiro também contribuem, mas em menor proporção.
\end{abstract}

PALAVRAS Chave: Parâmetros físico-químicos, Afluentes do Solimões, Águas pretas, Isótopos de Sr, Diagramas de estabilidade.

\section{The Solimões river hydrochemistry between Manacapuru and Alvarães - Amazonas - Brazil}

\begin{abstract}
The present study evaluated the physical and chemical characteristics of the water of the rivers Solimóes, Purus and their tributaries, collected in November of 2004 in the State of Amazonas between the cities of Manacapuru and Alvarães and Anamã and Pirarauara. Physical-chemical analyses (temperature, $\mathrm{pH}$, electrical conductivity, turbidity, $\mathrm{Ca}^{2+}, \mathrm{Na}^{+}, \mathrm{K}^{+}, \mathrm{Mg}^{2+}, \mathrm{HCO}_{3}^{-}$, $\mathrm{SO}_{4}^{2-}, \mathrm{Cl}^{-}$), and trace-elements (Li, B, Al, Sc, V, Cr, Mn, Fe, Co, Cu, Zn, As, Se, Rb, Sr, Mo, Cd, Sb, Cs, Ba, Pb, La, Ce e U) and $\mathrm{Sr}$ isotopes were accomplished. The analyzed parameters and the chemical composition show that the waters of the rivers and tributaries of the central region of the Amazonia are chemically distinct between them. The white waters of Solimóes are calcium-bicarbonate and of Purus are bicarbonates, and the respectives tributaries are sodium-potassium-bicarbonate and sodium-potassium-sulphate. This causes the white poorly acided waters to the neutrals and more conductives, while the black ones are less mineralized, more acids, mainly of Purus. The $\mathrm{Ba}, \mathrm{Sr}, \mathrm{Cu}, \mathrm{V}$ e As in highest levels differentiate the Solimóes white waters of the Purus's waters, well as the tributaries of the first related to the second. This complex of caracteristics indicates that the Solimôes as the Purus and their respectives tributaries are submitted to geological/ambiental distinct conditions. The influence of arrives in port of sediments of Andes is diluted at the currency of basin of the Solimóes and it reflects on formation of fertile valleys Solimóes and Purus. By another view, the crust rocks, represented by the Shields of the Guianas and Brasileiro both contribute, but in a reduced proportion.
\end{abstract}

KEYwORDS: Physical and chemical parameters, Tributaries of the Solimóes and Purus, Black water, Isotopes of Sr, Diagrams of stability.

\footnotetext{
1 Universidade Federal do Amazonas, Departamento de Geociências. Av. General Rodrigo Otávio Jordão Ramos, 3000 - Japiim. CEP: $69077-000$ Manaus, AM. e-mail: mireidequeiroz@ yahoo.com.br

2 Universidade Federal do Amazonas, Departamento de Geociências. Av. General Rodrigo Otávio Jordão Ramos, 3000 - Japiim. CEP: $69077-000$ Manaus, AM. e-mail: ahorbe@ ufam.edu.br

3 Université Paul Sabatier Institut de Recherche pour lê Développement (IRD), Laboratoire des Mécanismes de Transfert en Géologie (LMTG), 38 rue dês trente-six ponts, 31400 , Toulouse.e-mail: seyler@unb.br

${ }^{4}$ Universidade Federal do Pará, Instituto de Geociências. Rua Augusto Correia, 1 - Guamá. CEP: 66075-110 Belém, PA. e-mail: candido@ufpa.br
} 


\section{INTRODUÇÃO}

Os rios cujo, ambiente natural ainda está preservado, mostram relaçáo entre a hidroquímica e o ambiente por onde percolam (p. ex.: Sioli \& Klinge, 1962; Sioli, 1968; Gibbs, 1970; Stallard \& Edmond, 1983, 1987; Junk \& Furck, 1985; Viers et al., 2000; Tosiani et al., 2004; Berger \& Forberg, 2006; Hren et al., 2007). Alguns elementos podem ser relacionados diretamente com tipos de rochas, por exemplo $\mathrm{Mg}^{2+}, \mathrm{Ca}^{2+}$, $\mathrm{Sr}, \mathrm{HCO}_{3}^{-}$e $\mathrm{SO}_{4}^{2-}$ têm relação com carbonatos e gipsita; $\mathrm{K}^{+}$, $\mathrm{B}$ e $\mathrm{Na}^{+}$com illita; Si com bentonita e fontes termais; $\mathrm{Na}^{+}$e $\mathrm{Cl}^{-}$com evaporitos; Fe e B com glauconita. Outros como U, $\mathrm{PO}_{4}^{3-}, \mathrm{F}^{-}, \mathrm{NO}_{3}{ }_{3}^{-} \mathrm{Ni}, \mathrm{Cu}$ e $\mathrm{Zn}$ não mostram essa mesma relação (Stallard \& Edmond, 1983). Influências da vegetação são observadas nos rios de águas pretas que apresentam grande quantidade de matéria orgânica dissolvida, o que os torna mais ácidos (Sioli, 1985).

Sioli \& Klinge (1962), pioneiros no estudo dos rios da Amazônia, os classificaram em três categorias: águas brancas, pretas e claras. As brancas são típicas dos rios Solimôes, Amazonas, Madeira, Purus entre outros, possuem elevada quantidade de material em suspensão e sais dissolvidos provenientes dos Andes e da erosáo dos sedimentos encontrados ao longo das bacias de drenagens. $\mathrm{O} \mathrm{pH}$ é

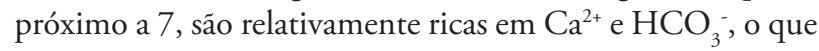
as classificam como carbonatadas (Sioli, 1968; Konhauser et al., 1994; Gaillardet, et al., 1997). As águas pretas, cujo principal representante é o rio Negro, provém da drenagem dos escudos das Guianas e Brasileiro. Sua cor é resultado de substâncias fúlvicas e húmicas dissolvidas, têm altos conteúdos de $\mathrm{Na}^{+}$e K+, o que acarreta menor $\mathrm{pH}(4,0)$ que as brancas (Furch, 1984; Walker, 1987; Forti et al., 1991). As claras (rios Tapajós e Xingu) são oriundas da Amazônia Central, que em virtude do relevo mais regular, oferece menor taxa de erosão. São límpidas, apresentam características químicas de transiçâo entre os dois tipos anteriores e o $\mathrm{pH}$ varia entre 4,5 e 7,0 (Sioli, 1960; Stallard \& Edmond, 1983).

Apesar dos rios Solimôes, Amazonas e Negro já terem sido alvo de vários estudos, a influência da diversidade geológica e de ambientes na Amazônia (terra firme, várzeas, igapós e lagos) na composição química dos rios ainda é pouco conhecida. Estudos nos pequenos tributários dos grandes rios da Amazônia mostram que há relação direta entre o ambiente e a química das águas (Santos \& Ribeiro, 1988; Cunha, 2000; Horbe et al., 2005). Portanto, são eles que melhor permitem avaliar a influência do ambiente na sua composição. Com vista a contribuir nesse tema, foram selecionados afluentes dos rios Solimóes e Purus no Estado do Amazonas, localizados na porçấo central do Amazonas (Fig. 1).

\section{CONTEXTO FISIOGRÁFICO E GEOLÓGICO}

A cobertura vegetal é de floresta densa (Radambrasil, 1978). O clima na região é do tipo tropical quente e úmido, com temperatura média anual de $27^{\circ} \mathrm{C}$, durante quase todo o ano, com sensível diminuiçáo de amplitude térmica no período das chuvas. Geralmente, de junho a agosto, devido às freqüentes entradas de frentes frias de origem polar, ocorre o fenômeno denominado friagem com maior influência no oeste da Amazônia e relativa diminuição da temperatura durante poucos dias (Nimer, 1979). Embora as massas de ar sejam em geral secas, é uma regiâo bastante úmida devido à alta taxa de evapotranspiração.

É relevante destacar o alto índice pluviométrico, com média de $2100 \mathrm{~mm} / \mathrm{ano}$, com o máximo de janeiro a maio quando tem-se o inverno regional e mínimo no verão amazônico de julho a outubro (Cáuper, 2000). A umidade relativa do ar é bastante elevada, apresenta nos meses mais chuvosos 80 a $90 \%$ e na estiagem atinge o mínimo de $75 \%$. $\mathrm{O}$ relevo tem pouca influência no clima, pois a maior parte do território tem altitude inferior a 200 metros (Cunha \& Appi, 1990).

As rochas sedimentares das Formaçôes Solimóes, Içá e sedimentos quaternários são as unidades geológicas que influenciam diretamente a química das bacias de drenagem dos afluentes dos rios Solimóes e Purus, apesar da forte influência dos sedimentos em suspensão em grande parte provenientes da erosão dos Andes. A Formação Solimôes é constituída de arenitos e siltitos de idade miocena de origem marinha (Nogueira et al., 2003). Segundo Horbe et al. (2007) quartzo, caolinita, illita, hematita+goethita e muscovita são os minerais principais e na sua composição química predominam $\mathrm{SiO}_{2}, \mathrm{Al}_{2} \mathrm{O}_{3}$ seguidos de $\mathrm{Fe}_{2} \mathrm{O}_{3}, \mathrm{~K}_{2} \mathrm{O}, \mathrm{CaO}, \mathrm{MgO}$ e $\mathrm{Na}_{2} \mathrm{O}$. Sobreposta ocorre a Formaçấo Içá, se estende do alto Solimóes até próximo a Manaus, é formada de arenitos e arenitos argilosos fluviais rosados e esbranquiçados, siltitos e argilitos friáveis amarelados, avermelhados, localmente ferruginizados que se assentam em discordância com a Formaçấo Solimóes (Maia et al., 1977).

Os sedimentos quaternários são genericamente, divididos em Quaternário Antigo e Recente, representam respectivamente as planícies aluviais e ilhas/barras dos rios de água branca da Amazônia. Os depósitos sedimentares recentes da calha do rio Solimóes-Amazonas são compostos por arenitos e siltitos constituídos de quartzo, caulinita, K-feldspato, plagioclásio, mica, hematita, fragmentos de rochas sedimentares (siltitos e arenitos), metamórficas (xistos) e vulcânicas, além de raros fragmentos de rochas carbonáticas (Franzinelli \& Potter, 1989). 


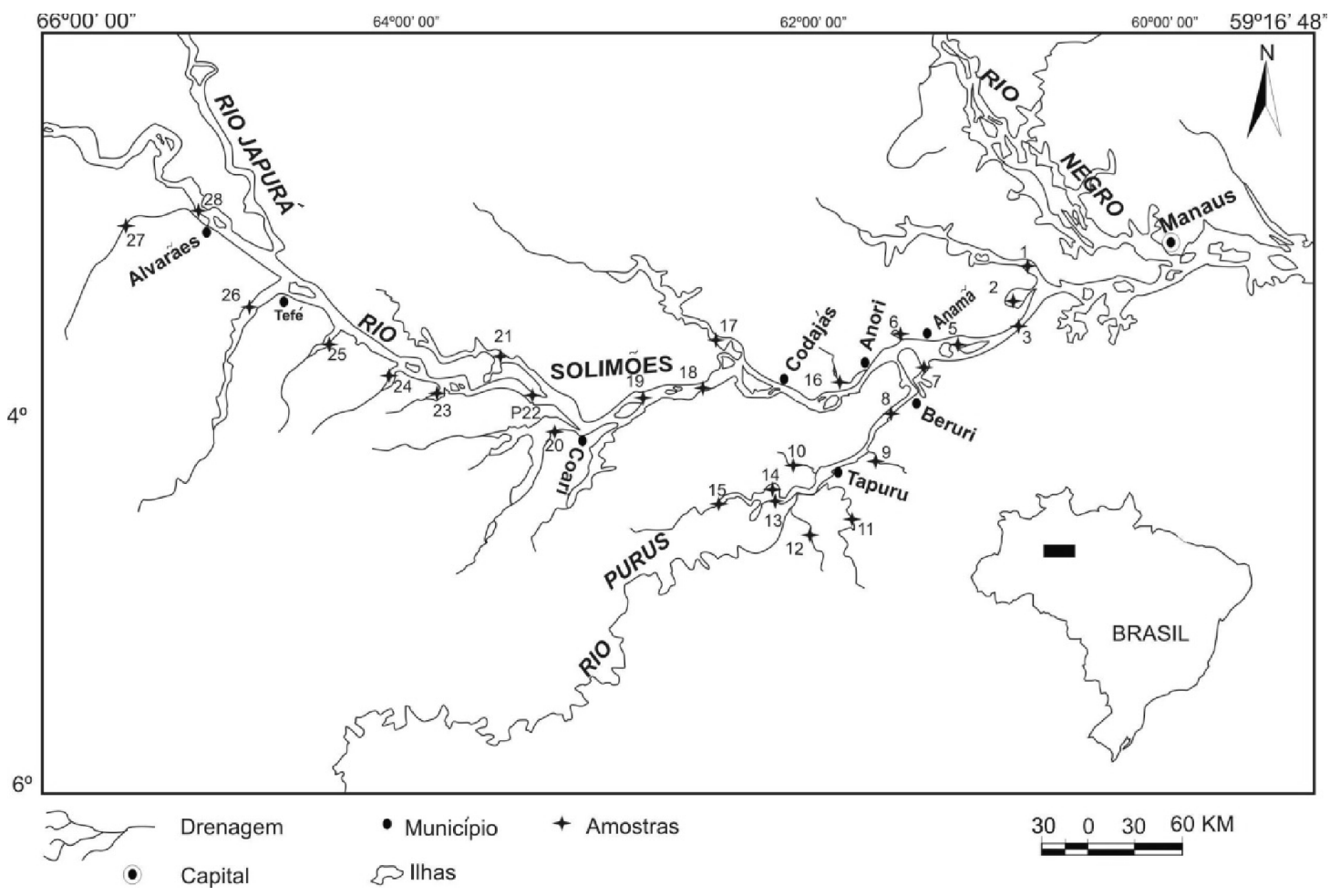

Figura 1- Mapa de localização das amostras coletadas.

\section{MATERIAL E MÉTODOS}

Foram coletadas oito amostras de água ao longo dos rios Solimóes e Purus e dezenove em seus principais afluentes na porção central do Amazonas em novembro de 2004, no fim do período de estiagem, quando o rio atinge seu nível mais baixo (Fig. 1). As amostras foram retiradas de jusante para montante, aproximadamente 15 centímetros de profundidade, coletadas em garrafas de polietileno de $1 \mathrm{~L}$ previamente desmineralizadas com solução de ácido nítrico $\left(\mathrm{HNO}_{3}^{-}\right)$a $25 \%(\mathrm{v} / \mathrm{v})$, lavadas com água deionizada e secas. Durante a amostragem os recipientes foram lavados três vezes com a própria amostra. No local da coleta foram analisados temperatura, $\mathrm{pH}$, condutividade elétrica (potenciometria), turbidez (turbidimetria), $\mathrm{HCO}_{3}{ }^{-} \mathrm{e} \mathrm{Cl}^{-}$(titulometria) uma vez que esses parâmetros se modificam rapidamente. As amostras foram filtradas com membrana de celulose de 0,45 $\mu \mathrm{m}$ e submetidas a análises de $\mathrm{SO}_{4}^{2-}$ (fotometria) no laboratório de Geoquímica da Universidade Federal do Amazonas. As demais amostras foram acidificadas com $\mathrm{HNO}_{3}$ bidestilado para posterior análise de $\mathrm{Ca}^{2+}, \mathrm{Na}^{+}, \mathrm{K}^{+}, \mathrm{Mg}^{2+}, \mathrm{Fe}, \mathrm{Al}, \mathrm{Zn}, \mathrm{Mn}$, $\mathrm{Ba}, \mathrm{Sr}, \mathrm{Cu}, \mathrm{B}, \mathrm{V}, \mathrm{Cr}, \mathrm{Co}, \mathrm{As}$, Se e Sb (espectrometria via ICPMS) no Laboratoire des Mécanismes de Transfert en Géologie (LMTG) - Université Paul Sabatier - Toulouse - França.

Os valores encontrados para $\delta{ }^{87} \mathrm{Sr}$, foram calculados com auxílio da fórmula: $\delta{ }^{87} \mathrm{Sr}=\left\{\left[\left({ }^{87} \mathrm{Sr} /{ }^{86} \mathrm{Sr}\right)_{\mathrm{a}} /\left({ }^{87} \mathrm{Sr} /{ }^{86} \mathrm{Sr}\right)_{\mathrm{am}}\right]-1\right\}$ x 1000 onde $\left({ }^{87} \mathrm{Sr} /{ }^{86} \mathrm{Sr}\right){ }_{\text {a }}$ é a razão isotópica da amostra e $\left({ }^{87} \mathrm{Sr} /{ }^{86} \mathrm{Sr}\right)$ am é a razão isotópica da água do mar atual $(0,70920)$. As análises foram realizadas no Laboratório de Geologia Isotópica do Centro de Geociências da UFPA - Pará-Iso em Belém.

Os resultados foram submetidos à análise estatística multivariada por componentes principais (Statística 6.1) com o objetivo de reduzir os parâmetros analisados (variáveis) e as amostras a um conjunto mais significativo. Esta técnica, que possibilita avaliar a inter-relação existente entre as variáveis (parâmetros) e as amostras, gera fatores e escores que representam à variância dos dados, ou seja, o grau de correlação ou significância. A análise multivariada abrangeu duas etapas: na primeira, obtiveram-se os escores de todas as variáveis; posteriormente, nos dois primeiros fatores, foram selecionadas aquelas maiores que 0,6 para determinar os escores das amostras, também nos dois primeiros fatores. Esses escores foram representados em gráfico para melhor visualização.

\section{RESULTADOS E DISCUSSÃO}

\section{PARÂMETROS FÍSICOS}

As temperaturas das águas variaram entre $28,2{ }^{\circ} \mathrm{C}$ e $34,5^{\circ} \mathrm{C}$ e não houve diferença em termos de cor, mas foi 
observado que as coletadas entre às 10 e 16 horas apresentaram temperaturas acima de $30^{\circ} \mathrm{C}$ (Tab. 1), em funçáo da alta incidência da radiação solar.

As águas dos rios Solimôes e Purus, classificadas como brancas, são fracamente ácidas a neutras $(6,5-7,0)$, enquanto as pretas são as mais ácidas, especialmente as dos afluentes do rio Purus (entre 5,3 e 6,7), enquanto as dos afluentes do Solimôes tendem a ser mais básicas $(5,9$ - 7,2) assemelhandose as brancas destes (Tab. 1). A maior acidez nas águas pretas, conforme relatado por diversos autores (Sioli \& Klinge, 1962; Sioli, 1968; Thurman, 1985; Starllard \& Edmond, 1987), é devido à decomposiçấo da matéria orgânica do solo em ácidos orgânicos (ácidos húmicos e fúlvicos), que são lixiviados para as águas. Esses ácidos, que têm em sua estrutura grupos carboxílicos $(-\mathrm{COOH})$ e hidroxílicos $(-\mathrm{OH})$, se dissociam e liberam na água íons $\mathrm{H}^{+}$, o que reduz o $\mathrm{pH}$ do meio. $\mathrm{O} \mathrm{pH}$ das águas dos rios da Amazônia também tem relação, até certo grau, com o ambiente geológico (Starllard \& Edmond, 1987), pois os minerais silicatados influenciam, juntamente com as espécies de $\mathrm{CO}_{2}$, as reaçóes do meio aquoso e conferem forte poder de tamponamento nas águas. Portanto, enquanto a matéria orgânica tende a acidificar a água, a geologia e os sedimentos em suspensão contribuem para manter o $\mathrm{pH}$ próximo a neutralidade, pois a dissolução dos silicatos por hidrólise consome íons $\mathrm{H}^{+}$e eleva o $\mathrm{pH}$ das águas.

A condutividade elétrica é mais elevada na águas brancas do rio Solimôes (média $98,8 \mu \mathrm{S} \mathrm{cm} \mathrm{cm}^{-1}$ ), seguida pelo Purus $\left(49,30 \mu \mathrm{S} \mathrm{cm}{ }^{-1}\right)$ e pelas águas pretas dos afluentes dos rios

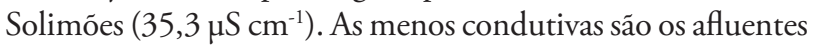
do Purus $\left(26,2 \mu S \mathrm{~cm}^{-1}\right)$ (Tab. 1).

Tabela 1 - Parâmetros físico-químicos das águas analisadas.

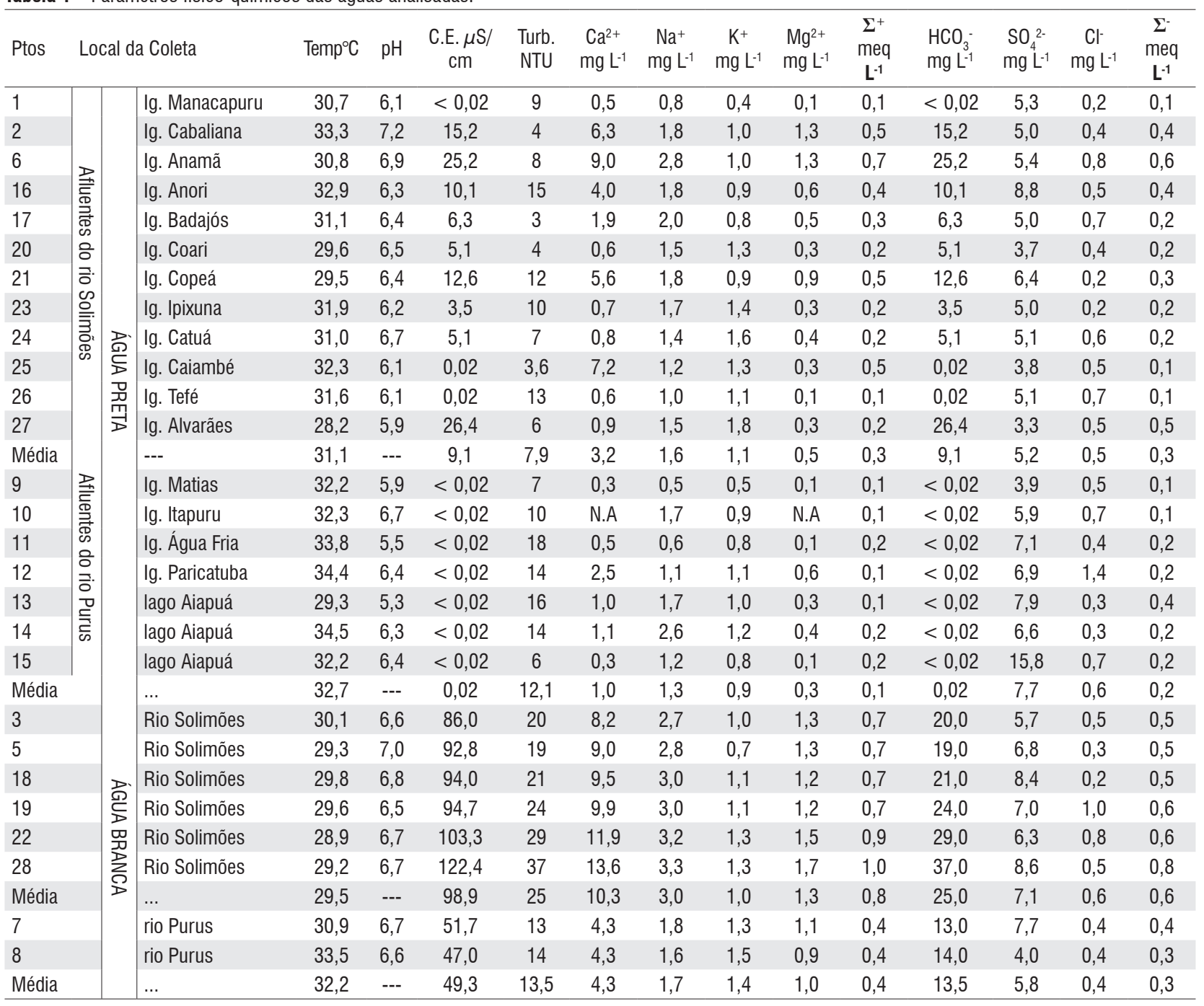

Temp.: Temperatura; C.E.:Condutividade elétrica; Turb.: Turbidez; ig.: igarapé; N.A.: Não Analisado.

Limite de detecção dos elementos analisados na tabela $1: \geq 0,02 \mathrm{mgL}^{-1}$. 


\section{PARÂMETROS QUÍMICOS}

$\mathrm{Na}$ somatória da carga total dissolvida, com erro inferior a 3\%, há predominância dos cátions sobre os ânions, especialmente no rio Solimóes $\left(4,7 \mathrm{meq} \mathrm{L}^{-1}\right)$, bem como nos seus afluentes $\left(3,9 \mathrm{meq} \mathrm{L}^{-1}\right)$ quando comparados aos do Purus (1,0 meq L $\left.{ }^{-1}\right)$ (Tab. 1). Segundo Appelo et al. (2005) a diferença no balanço de carga de $2 \%$ é inevitável e às vezes um erro de até $5 \%$ deve ser aceito.

A somatória dos cátions e de ânions é maior nas águas brancas, enquanto nas pretas é mais elevada nos afluentes do Solimóes que nos do Purus e em ambos há tendência de aumento para montante (Tab. 1). Dentre os cátions, predomina o $\mathrm{Ca}^{2+}$ nas brancas e este se alterna com o $\mathrm{Na}^{+}$nas pretas. Nas águas brancas esses dois cátions representam $64 \%$ da carga total, enquanto nas pretas perfazem $42 \%$ (Tab. 1). $\mathrm{O} \mathrm{HCO}_{3}$ é o ânion mais abundante, exceto nos afluentes do Purus onde predomina $\mathrm{SO}_{4}^{2-}$ e $\mathrm{HCO}_{3}^{-}$está abaixo do limite de detecção $\left(<0,02 \mathrm{mg} \mathrm{L}^{-1}\right)$ (Tab. 1). Nos afluentes do Solimôes, também há exceçôes, no igarapé Manacapuru predomina o $\mathrm{SO}_{4}^{2-}$ seguido do $\mathrm{Cl}^{-}$.

\section{ELEMENTOS-TRAÇO}

Dentre os elementos-traço analisados (Li, B, Al, Sc, V, Cr, $\mathrm{Mn}, \mathrm{Fe}, \mathrm{Co}, \mathrm{Cu}, \mathrm{Zn}, \mathrm{As}$, Se, Rb, Sr, Mo, Cd, Sb, Cs, Ba, Pb, $\mathrm{La}$, Ce e U) somente $\mathrm{Fe}, \mathrm{Al}, \mathrm{Zn}, \mathrm{Mn}, \mathrm{Ba}, \mathrm{Sr}, \mathrm{Cu}, \mathrm{B}, \mathrm{V}, \mathrm{Cr}$, $\mathrm{Co}$, As, Se e Sb apresentam valores significativos, contudo há variaçôes acentuadas (Tab. 2). O Fe é o elemento mais elevado (entre $53 \mu \mathrm{g} \mathrm{L}^{-1}$ e $917 \mu \mathrm{g} \mathrm{L}^{-1}$ ) sobre os demais, seguido do $\mathrm{Al}$ (entre 26 e $258 \mu \mathrm{g} \mathrm{L}^{-1}$ ). Ambos representam pelo menos $68 \%$ da carga dos elementos-traço nas águas brancas e $81 \%$ nas águas pretas, contudo, somente o $\mathrm{Al}$, mais elevado no Solimóes, permite diferenciar este rio das demais drenagens analisadas (Tab. 2). Segundo Robison (1981) e Förstner \& Wittmann (1983) os elevados teores de $\mathrm{Fe}$ e $\mathrm{Al}$ sáo atribuídos ao aumento nas condiçôes oxidantes do ambiente, pois na época da coleta das amostras ocorreram chuvas esparsas, que podem ter aumentado o teor de oxigênio dissolvido e a quantidade de material em suspensáo e alterado os processos de adsorção e co-precipitação dos elementos-traço.

Apesar das variaçôes acentuadas de elementos-traço, é possível diferenciar os afluentes de águas pretas do Solimóes dos do Purus (Tab. 1) pelo conteúdo, em geral mais elevado em Ba (4 a $\left.53 \mu \mathrm{g} \mathrm{L}^{-1}\right)$ e Sr $\left(6\right.$ a $\left.35 \mu \mathrm{g} \mathrm{L}^{-1}\right)$ nos do Solimôes, e de $\mathrm{Mn}$ nos afluentes mais a jusante deste $\left(7 \mathrm{a} 64 \mu \mathrm{g} \mathrm{L}^{-1}\right)$ e no Purus. Nas águas brancas a distribuição é mais homogênea e com o mínimo mais elevados em Mn (30 a $98 \mu \mathrm{g} \mathrm{L}^{-1}$ no Solimóes e 14 a $24 \mu \mathrm{g} \mathrm{L}^{-1}$ no Purus), bem como Ba (37 a 55 $\mu \mathrm{g} \mathrm{L} \mathrm{L}^{-1}$ no Solimóes e 34 a $35 \mu \mathrm{g} \mathrm{L} \mathrm{L}^{-1}$ no Purus) e Sr ( 50 a $78 \mu \mathrm{g}$ $\mathrm{L}^{-1}$ no Solimóes e 24 a $27 \mu \mathrm{g} \mathrm{L}^{-1}$ no Purus). Esses elementos são mais elevados nas águas brancas que nas pretas (Tab. 1).
$\mathrm{O} \mathrm{Cu}\left(0,9\right.$ a $\left.12,7 \mu \mathrm{g} \mathrm{L}^{-1}\right), \mathrm{B}\left(2,1\right.$ a $\left.9,6 \mu \mathrm{g} \mathrm{L}^{-1}\right), \mathrm{V}(0,4$ a 3,0 $\left.\mu \mathrm{g} \mathrm{L}^{-1}\right)$ e As $\left(0,2\right.$ a $\left.1,5 \mu \mathrm{g} \mathrm{L^{-1 }}\right)$ apesar das variaçóes, especialmente do segundo, tendem a serem mais elevados nas águas do Solimôes. Contudo, valores similares a estes ou mais altos de $\mathrm{Cu}$ são encontrados nos igarapés Cabaliana $(12,7 \mu \mathrm{g}$ $\left.\mathrm{L}^{-1}\right)$ e Copeá $\left(6,4 \mu \mathrm{g} \mathrm{L}^{-1}\right)$, afluentes do Solimóes (Tab. 2). No rio Purus as concentraçôes desses elementos se assemelham às águas pretas, enquanto $\mathrm{Zn}, \mathrm{Cr}$, $\mathrm{Co}$, Se e $\mathrm{Sb}$ quase não têm variaçóes entre os tipos de águas.

Quando comparados aos valores máximos permitidos (CONAMA 357/05) constata-se que, apesar do Fe estar acima do máximo permitido $\left(300 \mu \mathrm{g} \mathrm{L}^{-1}\right)$ na maioria das drenagens (Tab. 2), assim como o $\mathrm{Al}\left(200 \mu \mathrm{g} \mathrm{L} \mathrm{L}^{-1}\right)$, especialmente no ponto $22(258 \mu \mathrm{g} \mathrm{L}-1)$ do rio Solimóes, as demais características se mantém preservadas. Isso indica que não ocorreram modificaçôes significativas nos seus ambientes naturais. Os teores de $\mathrm{Fe}$ e $\mathrm{Al}$ encontrados neste estudo estão na mesma unidade de grandeza que os de Gaillardet et al. (1997), ElbazPoulichet et al. (1998), Mortati \& Probst (2003) e Seyler \& Boaventura (2003).

\section{COMPOSIÇÃO ISOTÓPICA DE ESTRÔNCIO}

A razão isotópica ${ }^{87} \mathrm{Sr} /{ }^{86} \mathrm{Sr}$ e $\delta{ }^{87} \mathrm{Sr}$ são mais elevadas nas águas brancas do rio Solimôes ( 0,708861 a 0,714461 e -0,369 a 7,418 \%o) e as mais baixos no rio Purus. Os afluentes do rio Purus têm razôes ${ }^{87} \mathrm{Sr} /{ }^{86} \mathrm{Sr}$ e $\delta^{87} \mathrm{Sr}$ entre 0,708685 a 0,713293 e $-0,726$ a $5,771 \%$, um pouco mais altas que os do rio Solimôes (0,708674 a 0,710980 e -0,025 a 2,509\%o) (Tab. 3). Destaca-se, ainda que a bacia do rio Purus é mais homogênea, com tendência a razóes menores no canal principal, exceto nos igarapés Itapuru e Paricatuba. Os da margem esquerda dos afluentes do rio Solimóes possuem ${ }^{87} \mathrm{Sr} /{ }^{86} \mathrm{Sr}$ mais baixas que os da direita, exceto Ipixuna e Caiambé, que são similares ao canal principal. Ao longo do Solimôes há tendência de redução da razão isotópica (de 0,714461 para 0,709098) para jusante.

Em relação à água do mar $(0,709211$ a 0,709241$)$ as razôes em geral, são mais elevadas (Fig.4), exceto nos igarapés Manacapuru, Cabaliana, Anamã, Copeá, Ipixuna, Caiambé, Alvarães (afluentes do Solimôes), Paricatuba (afluente do Purus) e parte do rio Solimões que são próximas da razão isotópica dos calcários (0,7082 - Allègre et al. 1996). Destacase que todas as amostras estáo abaixo da razáo isotópica dos rios Solimóes $(0,71319)$ e Negro $(0,71698)$ de Allègre et al. (1996).

\section{CLASSIFICAÇÃO E AMBIENTE GEOQUÍMICO}

Com o diagrama de Piper (Fig. 2) foi possível classificar as águas do rio Solimóes em cálcicas-bicarbonatadas e as do Purus em bicarbonatada, enquanto os seus afluentes de águas pretas, possuem tendência sódico-potássica-bicarbonatada e sódica-potássica-sulfatada, respectivamente. Essa tendência e a composição química como um todo das águas estudadas, é 


\begin{tabular}{|c|c|c|c|c|c|c|c|c|c|c|c|c|c|c|c|c|c|c|}
\hline Ptos & \multicolumn{3}{|c|}{ Local da Coleta } & $\mathrm{Fe}$ & $\mathrm{Al}$ & $\mathrm{Zn}$ & $\mathrm{Mn}$ & $\mathrm{Ba}$ & $\mathrm{Sr}$ & $\mathrm{Cu}$ & $B$ & $\mathrm{~V}$ & $\mathrm{Cr}$ & Co & As & Se & $\mathrm{Sb}$ & $\Sigma$ \\
\hline 1 & & \multirow{12}{*}{ 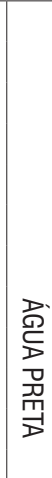 } & Ig. Manacapuru & 84 & 58 & 49 & 7 & 4 & 6 & 2,1 & 2,6 & 0,6 & 0,3 & 0,1 & 0,2 & 0,1 & 0,0 & 214 \\
\hline 2 & \multirow{10}{*}{ 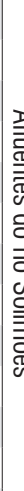 } & & Ig. Cabaliana & 408 & N.A & 180 & 34 & 34 & 33 & 12,7 & 4,9 & 1,8 & 1,3 & 0,3 & 1,0 & 0,2 & 0,5 & 7127 \\
\hline 6 & & & Ig. Anamã & 216 & 99 & 43 & 37 & 53 & 35 & 3,1 & 6,8 & 1,8 & 0,5 & 0,2 & 1,0 & 0,4 & 0,3 & 497 \\
\hline 16 & & & Ig. Anori & 356 & 82 & 27 & 45 & 24 & 29 & 3,8 & 3,4 & 1,8 & 0,5 & 0,3 & 1,1 & 0,1 & 1,2 & 575 \\
\hline 17 & & & Ig. Badajós & 189 & 27 & 11 & 24 & 19 & 17 & 3,0 & 3,6 & 0,8 & 0,3 & 0,2 & 0,7 & 0,1 & 0,4 & 296 \\
\hline 20 & & & Ig. Coari & 238 & 51 & 15 & 7 & 9 & 12 & 1,0 & 3,2 & 0,6 & 0,5 & 0,1 & 0,2 & N.A. & 0,4 & 338 \\
\hline 21 & & & Ig. Copeá & 677 & 188 & 39 & 64 & 32 & 32 & 6,4 & 4,6 & 2,0 & 1,5 & 0,6 & 1,0 & N.A. & 0,3 & 1048 \\
\hline 23 & & & Ig. Ipixuna & 917 & 71 & 27 & 11 & 12 & 20 & 1,4 & 3,6 & 1,1 & 1,0 & 0,2 & 0,3 & 0,1 & 0,7 & 1066 \\
\hline 24 & & & Ig. Catuá & 860 & 63 & 22 & 16 & 12 & 22 & 0,9 & 2,6 & 0,6 & 0,9 & 0,2 & 0,3 & 0,0 & 0,2 & 1001 \\
\hline 25 & & & Ig. Caiambé & 293 & 52 & 29 & 9 & 9 & 18 & 1,3 & 2,4 & 0,6 & 0,7 & 0,2 & 0,2 & 0,1 & 0,3 & 416 \\
\hline 26 & & & Ig. Tefé & 134 & 52 & 27 & 14 & 5 & 12 & 1,0 & 2,1 & 0,5 & 0,7 & 0,2 & 0,2 & 0,0 & 0,5 & 249 \\
\hline 27 & & & Ig. Alvarães & 386 & 84 & 23 & 12 & 14 & 25 & 2,3 & 3,9 & 0,9 & 0,8 & 0,4 & 0,2 & 0,1 & 0,3 & 5539 \\
\hline Média & \multirow{8}{*}{ 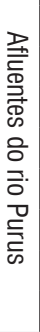 } & \multirow{18}{*}{ 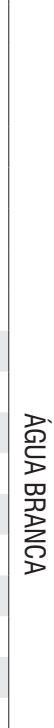 } & $\ldots$ & 396,5 & 75,2 & 41 & 23,3 & 19 & 22 & 3,3 & 3,6 & 1,1 & 0,8 & 0,2 & 0,5 & 0,1 & 0,4 & 1530,5 \\
\hline 9 & & & Ig. Matias & 108 & 51 & 32 & 12 & 2 & 7 & 1,7 & 2,2 & 0,4 & 0,5 & 0,3 & 0,2 & 0,1 & 0,2 & 218 \\
\hline 11 & & & Ig. Água Fria & 667 & 128 & 25 & 15 & 2 & 10 & 2,1 & 3,0 & 1,2 & 0,7 & 0,5 & 0,3 & N.A. & 0,9 & 857 \\
\hline 12 & & & Ig. Paricatuba & 532 & 96 & 25 & 64 & 15 & 30 & 2,1 & 3,2 & 1,2 & 0,8 & 0,6 & 0,7 & N.A. & 2,3 & 773 \\
\hline 13 & & & lago Aiapuá & 474 & N.A. & 29 & 43 & 8 & 18 & 3,1 & 5,2 & 1,2 & 0,6 & 0,5 & 0,6 & 0,7 & 0,5 & 584 \\
\hline 14 & & & lago Aiapuá & 367 & 71 & 22 & 28 & 15 & 17 & 2,1 & 6,5 & 1,0 & 0,6 & 0,3 & 0,6 & 0,2 & 0,4 & 532 \\
\hline 15 & & & lago Aiapuá & 429 & N.A & 19 & 26 & 4 & 9 & 1,7 & 3,3 & 0,8 & 0,3 & 0,4 & 0,2 & 0,1 & 2,2 & 496 \\
\hline Média & & & $\ldots$ & 429,5 & 86,5 & 25,3 & 31,3 & 8 & 15 & 2,1 & 3,9 & 1,0 & 0,6 & 0,4 & 0,4 & 0,2 & 1,1 & 577 \\
\hline 3 & & & rio Solimões & 265 & 98 & 30 & 43 & 37 & 50 & 4,0 & 6,7 & 1,7 & 0,7 & 0,3 & 0,9 & 0,3 & 0,2 & 538 \\
\hline 5 & & & rio Solimões & 195 & 121 & 26 & 30 & 37 & 55 & 3,9 & 9,5 & 1,7 & 0,7 & 0,3 & 1,0 & 0,5 & 0,2 & 482 \\
\hline 18 & & & rio Solimões & 592 & 170 & 22 & 56 & 40 & 55 & 4,0 & 7,1 & 2,4 & 0,9 & 0,5 & 1,2 & 0,0 & 0,4 & 952 \\
\hline 19 & & & rio Solimões & 547 & 156 & 25 & 74 & 45 & 57 & 5,1 & 7,2 & 2,4 & 1,6 & 0,2 & 1,2 & 0,1 & 0,8 & 923 \\
\hline 22 & & & rio Solimões & 758 & 258 & 43 & 98 & 55 & 69 & 5,2 & 8,2 & 3,0 & 1,4 & 0,8 & 1,5 & 0,3 & 0,9 & 1302 \\
\hline 28 & & & rio Solimões & 359 & 136 & 21 & 48 & 51 & 78 & 4,6 & 9,6 & 2,6 & 0,9 & 0,4 & 1,4 & 0,4 & 1,3 & 714 \\
\hline Média & & & $\ldots$ & 453 & 157 & 28 & 58 & 44 & 61 & 4,5 & 8,0 & 2,3 & 1,0 & 0,4 & 1,2 & 0,3 & 0,6 & 818,5 \\
\hline 7 & & & rio Purus I & 53 & 26 & 32 & 14 & 35 & 27 & 2,1 & 2,8 & 0,7 & 0,3 & 0,2 & 0,6 & 0,2 & 0,2 & 194 \\
\hline 8 & & & rio Purus II & 152 & 54 & 29 & 24 & 34 & 24 & 1,8 & 2,8 & 0,9 & 0,7 & 0,2 & 0,6 & 0,1 & 0,8 & 325 \\
\hline Média & & & $\ldots$ & 102,5 & 40 & 30,5 & 19 & 34,5 & 25,5 & 2,0 & 2,8 & 0,8 & 0,5 & 0,2 & 0,6 & 0,2 & 0,5 & 259,5 \\
\hline
\end{tabular}

ig.: igarapé; N.A.: Não Analisado.

compatível com os registrados por Stallard e Edmond, (1983, 1987); Konhauser et al. (1994); Gaillardet et al. (1997); Küchler et al. (2000) e Seyler e Boaventura (2003) em rios similares da Amazônia.

Dentre os elementos químicos analisados, a estatística multivariada por componentes principais $(\mathrm{CP})$ indicou, com base nas cargas mais elevadas $(\geq 0,6)$ do primeiro fator que a condutividade elétrica, $\mathrm{Ca}^{2+}, \mathrm{Mg}^{2+}, \mathrm{HCO}_{3}-\mathrm{SO}_{4}^{2-}, \mathrm{Cl}, \mathrm{Ba}$, $\mathrm{Sr}, \mathrm{Cu}, \mathrm{Sc}, \mathrm{V}$ e Co são as mais significativas para caracterizar as águas estudadas (Tab.4). Com base nessas variáveis, foram obtidos os escores das amostras e identificados grupos distintos. As águas pretas estão dispersas no segundo e terceiro quadrante e os igarapés Cabaliana (2) e Catuá (24) afluentes do Solimôes, com valores negativos de CP1 e CP2, localizam-se no quarto quadrante (Fig. 3). O Solimôes e Purus, localizados nos quadrantes 3 e 4 em conseqüência das cargas negativas do $\mathrm{CP} 2$, formam um grupo separado entre si e em relação a maioria das águas pretas, principalmente, devido a menor condutividade, $\mathrm{HCO}_{3}^{-}, \mathrm{Ca}^{2+}$ e Ba neste último (Fig. 3). A maior dispersão das águas pretas é indicativa da variabilidade dos ambientes percolados por essas águas (Stallard \& Edmond, 1983), especialmente, os afluentes do Solimóes, pois cobrem uma área maior que os do Purus (Fig. 1).

A relação entre as concentrações dos íons e isótopos nas águas de ambiente natural permitem inferir o tipo de rocha percolada e avaliar a mistura de águas que lixiviam regióes geologicamente distintas (Custódio \& Llamas, 1976; Stallard \& Edmond 1983; Faure, 1986). Dentre eles, $\mathrm{Na}^{+}$, $\mathrm{K}^{+} \mathrm{e} \mathrm{Cl}-$ são os mais representativos, pois são relativamente estáveis nas águas, facilmente analisados, têm tempo de residência mais elevada e não são afetados facilmente por variaçôes nas condiçôes de oxi-reduçáo do ambiente, como o sulfato (Gibbs, 1970, Stallard \& Edmond, 1983, 1987). Os valores encontrados nas razóes iônicas $(\mathrm{Na} / \mathrm{Cl}$ 0,60 - 21,0 e $(\mathrm{Na}+\mathrm{K}) / \mathrm{Cl} 2,35$ - 30,72 meq/L respectivamente) indicam à interação com rochas clásticas psamíticas e pelíticas, típicas 
Tabela 3 - Composição isotópica Sr nas águas estudadas.

\begin{tabular}{|c|c|c|c|c|c|c|}
\hline Pontos & Local da Coleta & & & $\begin{array}{l}\text { Razão isotópica } \\
{ }^{87} \mathrm{Sr} /{ }^{86} \mathrm{Sr}\end{array}$ & $\begin{array}{c}\delta^{87} \mathrm{Sr} \\
(\%)\end{array}$ & $\begin{array}{c}\mathrm{Sr} \\
\left(\mu \mathrm{g} \mathrm{L}^{-1}\right)\end{array}$ \\
\hline 1 & ig. Manacapuru (E) & \multirow{12}{*}{ 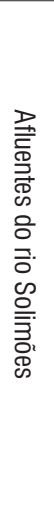 } & \multirow{12}{*}{ 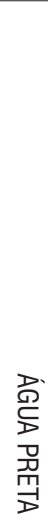 } & $0,709153 \pm 34$ & $-0,066$ & 5,51 \\
\hline 2 & ig. Cabaliana (E) & & & $0,709013 \pm 16$ & $-0,263$ & 32,52 \\
\hline 6 & ig. Anamã (E) & & & $0,709053 \pm 19$ & $-0,207$ & 35,05 \\
\hline 17 & ig. Badajós (E) & & & $0,709399 \pm 41$ & 0,28 & 16,63 \\
\hline 20 & ig. Coari (D) & & & $0,710980 \pm 15$ & 2,509 & 11,94 \\
\hline 21 & ig. Copeá (E) & & & $0,709182 \pm 68$ & $-0,025$ & 32,46 \\
\hline 23 & ig. Ipixuna (D) & & & $0,708961 \pm 01$ & $-0,336$ & 20,14 \\
\hline 24 & ig. Catuá (D) & & & $0,710691 \pm 09$ & 2,102 & 22,32 \\
\hline 25 & ig. Caiambé (D) & & & $0,708674 \pm 09$ & $-0,741$ & 17,94 \\
\hline 26 & ig. Tefé (D) & & & $0,710493 \pm 01$ & 1,823 & 11,89 \\
\hline 27 & ig. Alvarães (D) & & & $0,709067 \pm 19$ & $-0,187$ & 24,72 \\
\hline Média & & & & $0,709515 \pm 21$ & 0,444 & 21,69 \\
\hline 9 & ig. Matias (D) & \multirow{8}{*}{$\begin{array}{l}> \\
\text { D } \\
\text { 至 } \\
\text { क }\end{array}$} & \multirow{17}{*}{$\begin{array}{l}\text { 盆 } \\
\text { D } \\
\text { 叧 } \\
\text { 变 } \\
\text { D }\end{array}$} & $0,711244 \pm 58$ & 2,882 & 7,4 \\
\hline 10 & ig. Itapuru (D) & & & $0,710894 \pm 46$ & 2,388 & N.A \\
\hline 11 & ig. Água Fria (D) & & & $0,713118 \pm 62$ & 5,524 & 9,85 \\
\hline 12 & ig. Paricatuba (D) & & & $0,708685 \pm 63$ & $-0,726$ & 30,32 \\
\hline 13 & lago Aiapuá (D) & & & $0,713293 \pm 43$ & 5,771 & 18,27 \\
\hline 14 & lago Aiapuá (D) & & & $0,711426 \pm 14$ & 3,138 & 17,49 \\
\hline 15 & lago Aiapuá (D) & & & $0,711347 \pm 60$ & 3,027 & 8,77 \\
\hline Média & & & & $0,711429 \pm 49$ & 3,142 & 15,35 \\
\hline 3 & rio Solimões & & & $0,708938 \pm 26$ & $-0,369$ & 50,2 \\
\hline 5 & rio Solimões & & & $0,710659 \pm 38$ & 1,93 & 55,15 \\
\hline 18 & rio Solimões & & & $0,708861 \pm 64$ & $-0,478$ & 55,37 \\
\hline 19 & rio Solimões & & & $0,710698 \pm 67$ & 2,112 & 56,61 \\
\hline 22 & rio Solimões & & & $0,709219 \pm 19$ & 0,026 & 69,32 \\
\hline 28 & rio Solimões & & & $0,714461 \pm 81$ & 7,418 & 78,04 \\
\hline Média & & & & $0,710275 \pm 50$ & 1,770 & 61,48 \\
\hline 8 & rio Purus (D) & & & $0,711135 \pm 96$ & 2,728 & 24,39 \\
\hline Média & & & & $0,7111135 \pm 96$ & 2,728 & 24,39 \\
\hline
\end{tabular}

D.:direita; E.:Esquerda; N.A.: Não Analisado.
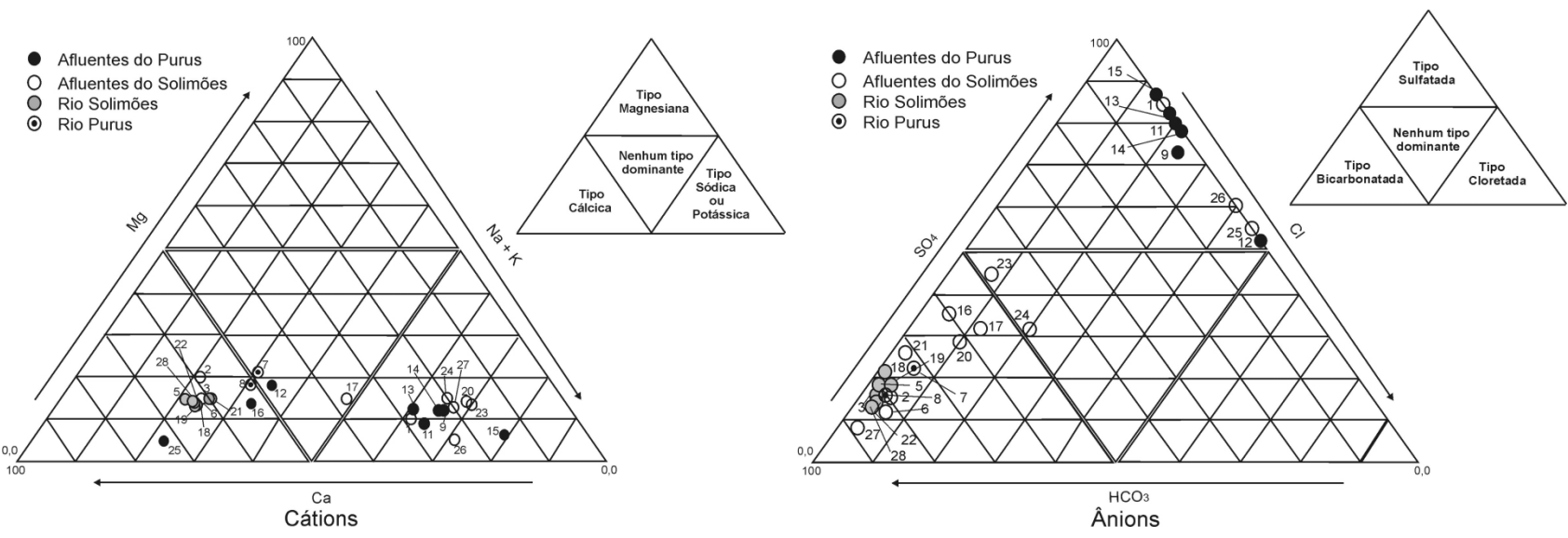

Figura 2 - Diagrama de Piper das águas estudadas. 


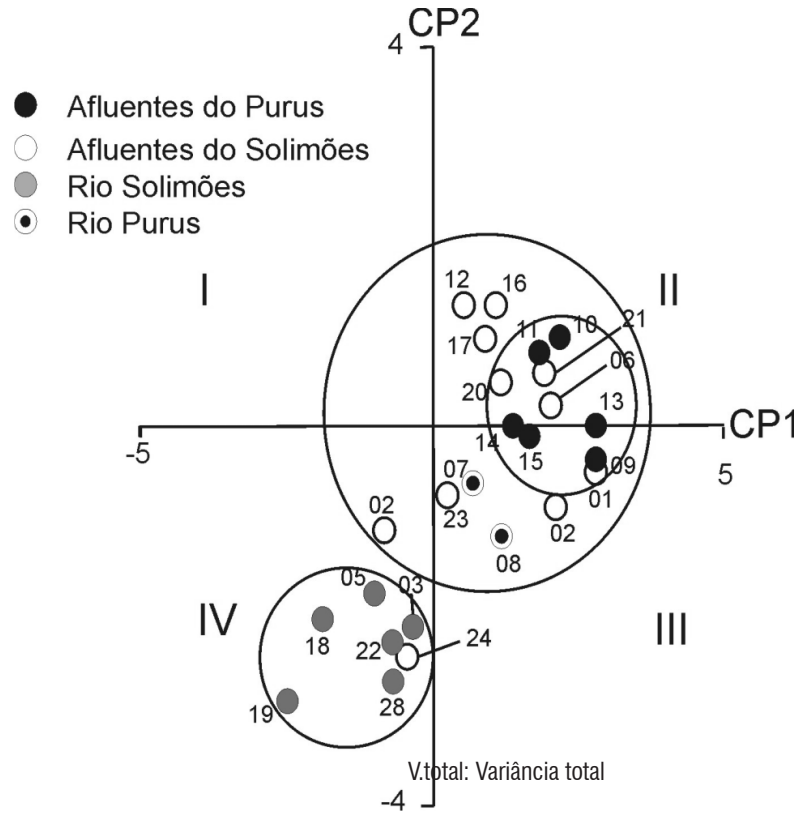

Tabela 4 - Análise de componentes principais das amostras de água.

\begin{tabular}{lccccc}
\hline Variáveis & $\mathrm{CP} 1$ & $\mathrm{CP} 2$ & Variáveis & $\mathrm{CP} 1$ & $\mathrm{CP2}$ \\
\hline $\mathrm{pH}$ & $-0,14$ & 0,66 & $\mathrm{~B}$ & $-0,39$ & $-0,22$ \\
$\mathrm{C} . \mathrm{E}$ & $-0,69$ & 0,38 & $\mathrm{Sc}$ & $-0,79$ & 0,31 \\
\hline Transp. & 0,01 & 0,48 & $\mathrm{~V}$ & $-0,68$ & $-0,56$ \\
$\mathrm{Ca}^{2+}$ & $-0,61$ & 0,64 & $\mathrm{Cr}$ & $-0,40$ & 0,57 \\
$\mathrm{Na}^{+}$ & $-0,46$ & 0,15 & $\mathrm{Co}$ & $-0,65$ & $-0,49$ \\
$\mathrm{~K}^{+}$ & $-0,57$ & $-0,59$ & $\mathrm{Ce}$ & $-0,31$ & $-0,35$ \\
$\mathrm{Mg}^{+}$ & $-0,92$ & 0,25 & $\mathrm{La}$ & $-0,32$ & 0,29 \\
$\mathrm{HCO}_{3}{ }^{-}$ & $-0,92$ & 0,25 & $\mathrm{U}$ & $-0,24$ & 0,24 \\
$\mathrm{SO}_{4}{ }^{2-}$ & $-0,75$ & 0,18 & $\mathrm{As}$ & $-0,38$ & 0,58 \\
$\mathrm{PO}_{4}{ }^{3-}$ & $-0,65$ & $-0,62$ & $\mathrm{Mo}$ & $-0,23$ & $-0,88$ \\
$\mathrm{Cl}^{-}$ & $-0,64$ & $-0,13$ & $\mathrm{Cd}$ & $-0,19$ & 0,09 \\
$\mathrm{Zn}$ & 0,21 & 0,04 & $\mathrm{Cs}$ & $-0,00$ & $-0,10$ \\
$\mathrm{Ba}$ & $-0,74$ & $-0,19$ & $\mathrm{Sb}$ & $-0,06$ & $-0,60$ \\
$\mathrm{Sr}$ & $-0,89$ & $-0,17$ & $\mathrm{~Pb}$ & $-0,01$ & $-0,19$ \\
$\mathrm{Cu}$ & $-0,88$ & 0,17 & V.total & $47 \%$ & $24 \%$ \\
\hline
\end{tabular}

Figura 3 - PC1 versus PC2 das amostras de águas.

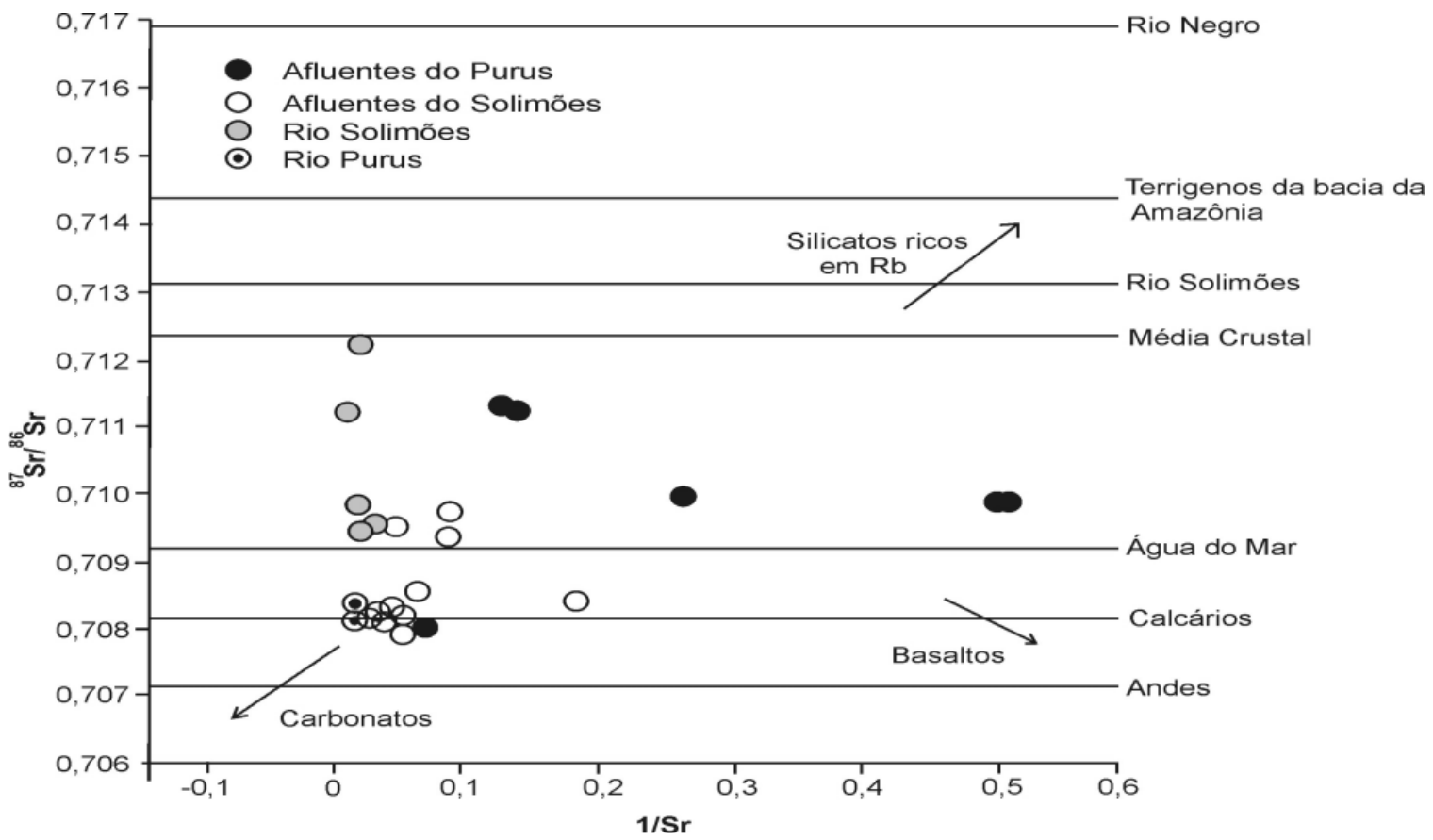

Figura $4-{ }^{87} \mathrm{Sr} /{ }^{86} \mathrm{Sr}$ vs 1/Sr das amostras de água em relação a rochas e sedimentos (Henderson, 1984; Faure, 1988 e Allègre et al. 1996) 


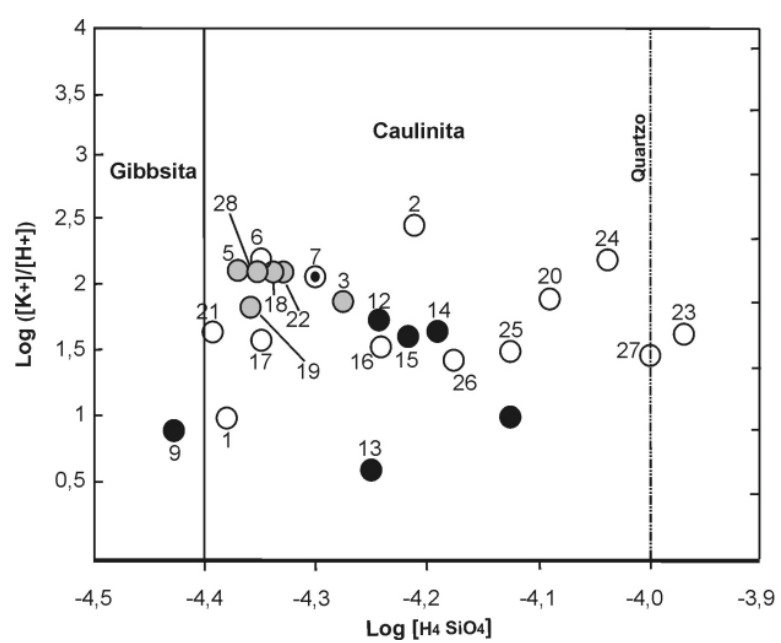

da bacia do rio Solimóes. Contudo, as variaçóes encontradas indicaram que tanto o Solimóes, como o Purus e os respectivos afluentes, estão submetidos a condiçóes geológicas/ambientais distintas.

Outro modo de determinar a influência do ambiente geológico e os processos geoquímicos é por meio de diagramas de atividade que descrevem o equilíbrio químico entre minerais e soluçóes aquosas (Drever, 1997). O diagrama $\log \left(\left[\mathrm{K}^{+}\right] /\left[\mathrm{H}^{+}\right]\right.$versus $\log \left[\mathrm{H}_{4} \mathrm{SiO}_{4}\right]$ (Fig. 5) elaborado para temperatura de $25^{\circ} \mathrm{C}$ mostra que as águas estão em equilíbrio com a caulinita e que são super saturadas em quartzo, minerais que predominam nos sedimentos da regiāo (Franzinelli \& Potter, 1989 e Horbe et al., 2007). Isto indica ambiente lixiviante, submetido a alta pluviosidade, compatível com o observado na Amazônia.

\section{CONCLUSÕES}

Os parâmetros analisados e a composição química mostram que as águas dos rios e igarapés da regiáo central da Amazônia são quimicamente distintas entre si. As águas brancas do Solimóes são cálcicas-bicarbonatadas e as do Purus bicarbonatadas, os respectivos afluentes são sódico-potássicobicarbonatados e sódico-potássico-sulfatados. Isso acarreta águas brancas fracamente ácidas a neutras e mais condutivas, enquanto as pretas são as mais ácidas. A somatória dos cátions e ânions é maior nas águas brancas e, conseqüentemente a condutividade, enquanto nas pretas essa relação é mais elevada nos afluentes do Solimóes que nos do Purus. Dentre os parâmetros analisados, $\mathrm{pH}$, condutividade, $\mathrm{Ca}^{2+}, \mathrm{Na}^{+}, \mathrm{K}^{+} \mathrm{e}$ Fe são os mais significativos na identificação das características das bacias. Desse modo, o Solimóes é marcado pelo maior teor

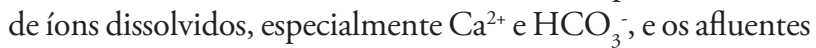
do Purus, por serem menos mineralizados, especialmente em direção a jusante. As águas pretas deste estudo, são distintas entre si e em relação ao rio Negro, possuem teores de elementos dissolvidos mais elevados que este, especialmente em álcalis.

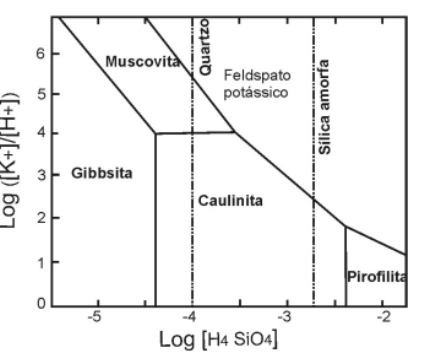

Afluentes do Purus

Afluentes do Solimões

Rio Solimões

Rio Purus
Figura 5 - Relação da estabilidade de alguns minerais no sistema $\mathrm{K}_{2} \mathrm{O}-\mathrm{Al}_{2} \mathrm{O}_{3}-\mathrm{SiO}_{2}-\mathrm{H}_{2} \mathrm{O}$ a $25^{\circ} \mathrm{C}$.

Portanto, a carga química reflete o meio ambiente lixiviante com alta pluviosidade da Amazônia e indica a variabilidade do ambiente geológico e hidrológico em que estão situadas, uma vez que não foi constatada atuação antrópica.

\section{AGRADECIMENTOS}

Os autores agradecem a Universidade Federal do Amazonas (UFAM) e a Coordenaçáo de Aperfeiçoamento de Pessoal de Nível Superior (CAPES), pela bolsa de mestrado do primeiro autor. Agradecem também a infra-estrutura do LMTG (Laboratoire des Mécanismes de Transfert en Géologie) Université Paul Sabatier - Toulouse - França.

\section{BIBLIOGRAFIA CITADA}

Allègre, C.J.; Dupré, B.; Négrel, P.; Giillardet, J. 1996. Sr-Nd$\mathrm{Pb}$ isotopes systematics in Amazon and Congo river sistems: Constrain about erosion proceses, Chemical Geology, 131: 93-112

Appelo, C.A.J.; Postma, D. 2005. Geochemistry, Growndwater and Pollution. Balkema, Brookfiekd, USA, 4-18pp.

Berger, L.; Forberg, B.R. 2006. Factors Controlling Hg levels in two predatoru fish species in the Negro River basin, Brasilian Amazon. Science of the total Environment, 367:451-459.

Cáuper, G.C.B. 2000. A influência das atividades de exploração de petróleo e os impactos ambientais e socioeconômicos na cidade de Coari. Dissertaçáo de Mestrado. Universidade Federal do Amazonas, Manaus, Amazonas. 146pp.

CONAMA (Conselho Nacional do Meio Ambiente). 2005. Resolução n. 357, de 17 de março de 2005. Brasília, DF.

Cunha, H.B.; Simões, C.A. 2000. Caracterização físico-químicas das águas do Rio Negro e seus tributários. In. IX Jornada de Iniciação Cientifica. Anais. Manaus- Amazonas, 325-329.

Cunha, F.B.; Appi, V.T. 1990. Controle geológico na definição de grandes domínios ambientais na planície Amazônica. Petrobrás/ Cenpes, 30-35.

Custódio, E.; Llamas, M.R.1976. Hidrologia subterrânea. Barcelona: Omega, 50pp. 
Elbaz-Poulichet, F.; Seyler, P.; Maurice-Bourgoin, L.; Guyot, J.L.; Dupré, C. 1998. Trace element geochemistry in the upper Amazon drainage basin (Bolivia). Mineralogical Magazine, 62: 418- 423.

Faure, G. 1986. Principles of Isotope Geology. New York, John Wiley \& Sons, 589pp.

Förstner, U.; Wittmann, G.T.W. 1983. Metal Pollution in the Aquatic Environment. Berlin, Springer-Verlag, 486pp.

Forti, M.C.; Melfi, A.J.; Amorin, P.R.N. 1991. Hidroquímica das águas de drenagem de uma pequena bacia hidrográfica no Nordeste da Amazônia (Estado do Amapá, Brasil): efeitos da sazonalidade. Geochimica Brasiliensis, 11(3): 311-340.

Franzinelli, E.; Potter, P.E. 1989. Areias recentes dos rios da Bacia Amazônica: Composiçôes Petrográfica, Textural e Química. Revista Brasileira de Geociências, 15: 213 - 220.

Furch, K. 1984. Water chemistry of the Amazon Basin: the distribution of chemical elements among freshwaters. In: Sioli, H. (ed.). The Amazon Limnology and landscape ecologyof a mighty tropical river and its basin. Junk, Dordrecht: 167-169.

Gaillardet, J.; Dupré, B.; Allègre, C.; Négrel, P. 1997. Chemical and Physical Denudation in the Amazon River Basin. Chemical Geology, 142: 141-173.

Gibbs, R.J. 1970. Water chemistry of Amazon river. Geochimica et Cosmochimica Acta, 36:1061-1066.

Drever, J.I. 1997. The Geochemistry of Natural Waters Surface and Groundwater Environments. Prentice Hall, 460pp.

Hren, M.T.; Chamberlain, C.P.; Hilley, G.E.; Blisniuk, P.M.; Bookhagen, B. 2007. Major ion chemistry of the Yarlung Tsangpo-Brahmaputra river: Chemical weathering, erosion, and $\mathrm{CO}_{2}$ consumption in the southern Tibetan plateau and eastern syntaxis of the Himalaya. Geochimica et Cosmochimica Acta, 71:2907-2935.

Horbe, A.M.C.; Gomes, I.L.F.; Miranda, S.A.F.; Silva, M.S.R. 2005. Contribuição à hidroquímica de drenagens no Município de Manaus - Amazonas. Acta Amazônica, 35:119-124.

Horbe, A.M.C.; Paiva, M.R.P.; Motta, M.B.; Horbe, M.A. 2007. Mineralogia e geoquímica dos perfis sobre sedimentos neógenos e quaternários da bacia do Solimóes na região de Coari Amazonas. Acta Amazônica, 37: 81-90

Junk, W.J.; Furch, K. 1985. Química da água e macrófitas aquáticas de rios e igarapés na bacia Amazônica e nas áreas adjacentes. Acta Amazônica, 10 (3): 611-633.

Konhauser, K.O.; Fyfe, W.S.; Kronberg, B.I. 1994. Multi-element chemistry of some Amazonian waters and soils. Chemical Geology, 111:155-175.

Külcher, I.L.; Miekeley, N.; Forsberg, B. 2000. A contribution to the chemical characterization of rivers in the rio Negro basin. Journal of Brazilian Chemical Society, 11:286-292.

Maia, R.G.N.; Godoy, H.K.; Yamaguti, H.S.; Moura, P.A.; Costa, F.S.F.; Holanda, M.A.; Costa, J.A. 1977. Projeto Carvão no alto Solimóes. Relatório Final, CPRM-DNPM, 137pp.

Mortatti, J.; Probst, J.L. 2003. Silicate rock weathering and atmospheric/soil $\mathrm{CO}_{2}$ uptake in the Amazon Basin estimated from river water geochemistry: seasonal and spatial variations. Chemical Geology, 197:177-196.

Nimer, E. 1979. Climatologia do Brasil. Rio de Janeiro: IBGE.

Nogueira, A.C.R.; Arai, M.; Horbe, A.M.C.; Horbe, M.A.; Silveira, R.R.; Silva, J.S.; Motta, M.B. 2003. A Influência Marinha nos Depósitos da Formação Solimốes na Região de Coari (Amazonas): Registro da Transgressão Miocênica na Amazônia Ocidental. VIII Simpósio de Geologia da Amazônia, sessão temática: Sedimentologia e Estratigrafia, CR-ROM.

RadamBrasil. 1978. Folha SB-20 Purus, Geologia, geomorfologia, solos, vegetaçâa e uso potencial da terra do Amazonas.

Robinson, G.D. 1981. Adsorption of $\mathrm{Cu}, \mathrm{Zn}$ and $\mathrm{Pb}$ near sulfide Deposites by hidrous Manganese-iron Coatings on stream Alluvium. Chemical Geology, 33:65-79.

Santos, U. M.; Ribeiro, M. N. G. 1988. A Hidroquímica do rio Solimôes - Amazonas. Acta Amazônica, 18 (3-4): 145 - 172.

Seyler, P., Boaventura, G.R. 2003. Distribution and partition of trace Metals in the Amazon Basin. Hydrological Processes, 13451361.

Sioli, H. 1960. Pesquisas limnológicas na regiāo da Estrada de Ferro de Bragança, Estado do Pará-Brasil. Boletim Técnico do Instituto Agronômico do Norte, (37):1-73.

Sioli, H.; Klinge, H. 1962. Solos, típicos de vegetação e águas na Amazônia. Amazoniana, 1:27-41.

Sioli, H. 1968. Hydrochemistry and Geology in the Brazilian Amazon Region. Amazoniana,3:267-277.

Stallard, R.F.; Edmond, J.M. 1983. Geochemistry of the Amazon. 2. The influence of geology and weathering environment on the dissolved load. Journal of Geophysical Research, 88:9671-9688.

Stallard, R.F.; Edmond, J.M. 1987. Geochemistry of the Amazon. 3. Weathering Chemistry and limits of dissolved inputs. Journal of Geophysical Research, 92: 8293-8302.

Thurman, E.M. 1985. Organic Geochemistry of Natural Waters. Boston, Martinus Nijhoffl Junk. 497 pp.

Tosiani, T.; Loubet, M.; Viers, J.; Valladon, M.; Tapia, J.; Marrero, S.; Yanes, C.; Ramirez, A.; Dupré, B. 2004. Major and trace elements in river-borne materials from the Cuyuni basin (southern Venezuela): evidence for organo-colloidal control on the dissolved load and element redistribution between the suspended and dissolved load. Chemical Geology, 211:305334.

Viers, J.; Dupré, B.; Braun, J.; Deerdt, S.; Angeletti, B.; Ngoupayou, J.N.; Michard, A. 2000. Major and trace element abundances, and strontium isotopes in the Nyong basin rivers-Cameroon: Constraints on chemical weathering processes and elements transport mechanisms in humid tropical environments. Chemical Geology, 169:211-241.

Walker, I. 1987. The biology of streams as part of Amazonian forest ecology. Experientiae, 73:279-287.

Recebido em 14/07/2008

Aceito em 29/12/2008 